Mediterránea Ser. Biol. (1988), n. ${ }^{\circ}$ 10. Pág. 29-44

\title{
ANÁLISIS DE LA DISTRIBUCIÓN DE GEOTRUPIDAE EN ÁREAS DEL OESTE ESPAÑOL: PROVINCIA DE CÁCERES
}

\author{
por \\ EDUARDO GALANTE PATIÑO ${ }^{1}$ y JESÚS RODRÍGUEZ ROMO ${ }^{1}$
}

\section{RESUMEN}

Se estudia la comunidad de Geotrupidae de la provincia de Cáceres, aportando datos de la distribución espacial y temporal de las especies que forman esta comunidad. También se profundiza en los hábitos de alimentación y biología de alguna de las especies estudiadas.

\section{SUMMARY}

The Geotrupidae guild of the Cáceres province are studied and the spacial and seasonal data of the species are given. Also some feed and biological habits of some of these species are given. Also some feed and biological habits of some of these species are adduced.

Palabras clave: Geotrupidae: distribución; área mediterránea.

\section{INTRODUCCIÓN}

Las especies de coleópteros coprófagos juegan un papel muy importante en el reciclaje rápido de la materia orgánica depositada por los herbívoros en forma de excrementos (FINCHER, HOWSON and BURTON, 1981). Gran parte de esta materia será rápidamente enterrada para servir de alimento a los escarabeidos (SCARABAEIDAE y GEOTRUPIDAE) adultos o bien a las futuras larvas.

Las especies de la familia GEOTRUPIDAE pueden tener gran importancia en la destrucción de heces y en los cambios fisicoquímicos que se producen en el medio, dado sus hábitos de alimentación y nidificación, así como su gran talla.

(1) Departamento de Zoología. Facultad de Biología. Universidad de Salamanca. 37071 SALAMANCA. 
Continuando con la investigación de comunidades de escarabeidos coprófagos en la Península Ibérica, se abordó el estudio de los mismos en un área poco conocida bajo el punto de vista entomológico como es la provincia de Cáceres. El conocimiento de los escarabeidos coprófagos de esta zona presentaba para nosotros gran interés tanto por su situación geográfica en plena región mediterránea, sometida a una clara influencia atlántica, así como por estar dedicada en su mayor parte a la actividad ganadera (ganado ovino y bovino fundamentalmente). Este hecho unido al conocimiento que ya teníamos de estos grupos de coleópteros en la provincia de Salamanca (GALANTE, 1981), que se encuentra separada de la de Cáceres por las estribaciones occidentales del Sistema Central (Sierras de Béjar, Peña de Francia y Gata) y que actúa como auténtica barrera natural en la distribución de diversas especies, dato que hemos podido comprobar a lo largo de la realización de este trabajo, nos indujo a elegir dicha zona como área de estudio.

Un hecho que se debe tener en cuenta al analizar los datos obtenidos, es que durante los meses estivales gran parte de la ganadería ovina y bovina del centro y sur de la provincia es trasladada a áreas montañosas, bien dentro de los límites provinciales, bien fuera de ellos, lo cual influye indudablemente en la distribución que presentan las especies cuyos imagos tienen mayor presencia y actividad en esa época del año. No obstante como ya conocemos por otras regiones y se confirma en este trabajo, los geotrúpidos serán los menos afectados por este hábito, ya que la mayor parte de las especies no presentan actividad durante el verano.

\section{ÁREA DE ESTUDIo}

La provincia de Cáceres está situada entre los $39^{\circ} 5^{\prime}$ y $46^{\circ} 28^{\prime}$ de latitud Norte y $4^{\circ} 56^{\prime}$ y $7^{\circ} 31^{\prime}$ longitud Oeste del meridiano de Greenwich, teniendo una extensión de $19.945 \mathrm{~km}$. En el límite Norte encontramos una barrera natural, constituida por las sierras más occidentales del Sistema Central: Sierra de Gata, Sierra de peña de Francia, Sierra de Bejar y Sierra de Gredos. En el sureste de la provincia se levantan las sierras de Guadalupe y Villuercas que constituyen el sector más occidental de los Montes de Toledo.

De acuerdo con GARCÍA y FORTEZA (1986), esta provincia acusa un clima de transición entre el continental de la meseta y el atlántico. El Sistema Central limita la penetración de los vientos fríos del Norte, y la altitud media, relativamente baja, unos $400 \mathrm{~m}$., facilita la llegada de los procedentes del Atlántico. No obstante, las diferencias de altitud y orientación de las sierras y valles dan lugar a climas bien diferenciados.

Por su situación la provincia de Cáceres tiene como vegetación predominante la esclerófila mediterránea, representada por las grandes formaciones de durilignosa de la encina (Quercus rotundifolia Lam.). Sus formaciones, los encinares, incluidos en la clase fitosociológica Querceta ilicis $\mathrm{Br}$. Bl., ya sean en estado puro, ya sean adehesados o mezcla con alcornoques (Quercus suber L.) o quejigos ( $Q$. faginea Lam), cubren gran parte de la superficie provincial, siendo la formación arbórea más importante.

La otra gran formación arbórea, que se encuentra bien representada en las sierras septentrionales y en menor proporción en el sudeste (Villuercas) y 
suroeste (Valencia de Alcántara), es un bosque caducifolio, constituido por la especie caducifolia o marcescente de montaña Quercus pyrenaica will. (roble melojo), en ocasiones sustituido por el nombre por el castaño (Castanea sativa Miller).

En el mapa de vegetación (Mapa 1) podemos observar estas dos grandes formaciones arbóreas, así como las distintas etapas de sustitución de éstas, y diversas formaciones arbóreas o arbustivas de menor importancia.

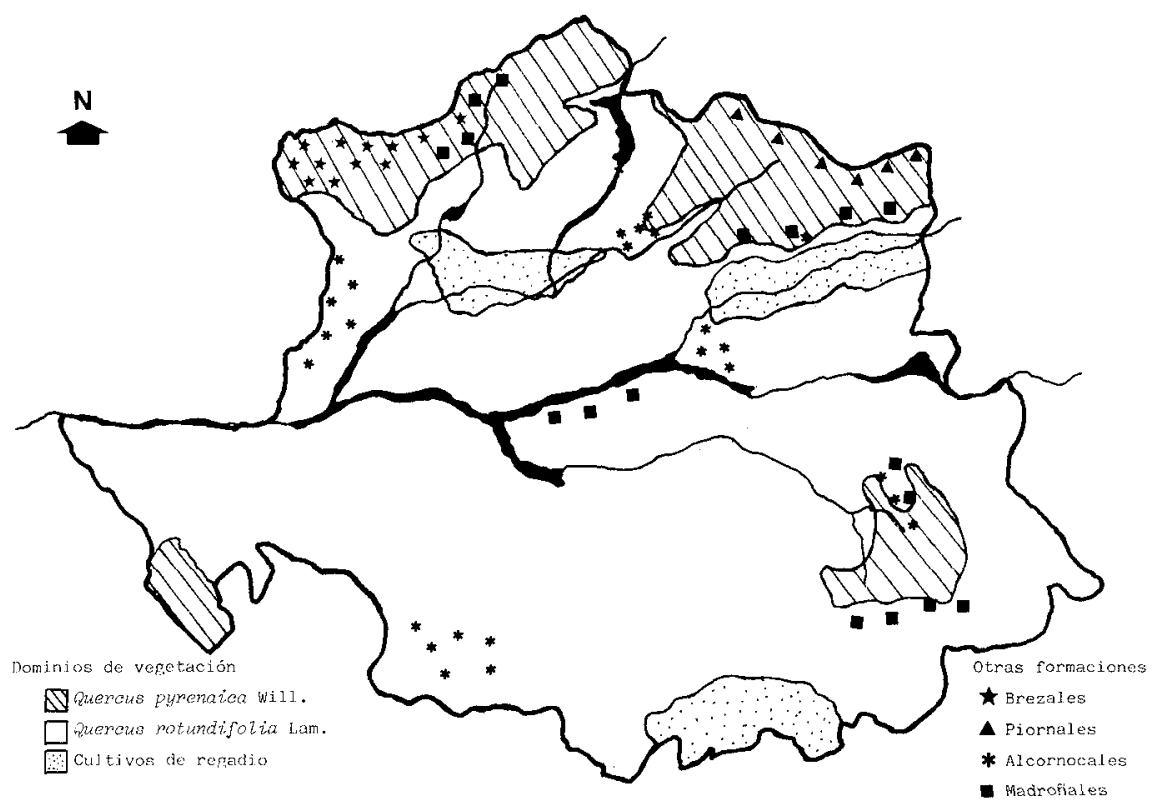

Mapa 1: Dominios de vegetación de la provincia de Cáceres.

\section{MATERIAL Y MÉTODOS}

Los muestreos han sido realizados durante los años 1980-85, aunque el mayor número de estos se llevó a cabo en los años 1980, 1982 y 1983. En los años 1984-85 se ha efectuado un número reducido, pero selectivo, de muestreos, que han servido para aumentar la información en cuanto a la distribución y actividad anual de las diferentes especies colectadas.

Se han prospectado 98 localidades repartidas por toda la provincia (Mapa 2), pero con mayor incidencia en el área septentrional, puesto que la riqueza ganadera es mayor en esta zona. Se han considerado como unidad de muestreo cada una de las visitas realizadas en una localidad, en una fecha determi- 
nada y recogiendo un tipo de excremento. En total se han efectuado 233 muestreos habiéndose colectado 268 individuos de la familia Geotrupidae. También ha sido incluido en este estudio el material que existía en el Departamento de Zoología de la Universidad de Salamanca.

Del conjunto de especies recolectadas, únicamente se ha estudiado el ritmo de actividad anual de los adultos en aquellos casos de que se disponía de un número representativo de individuos. Para calcular la actividad anual de cada especie se ha empleado un índice corregido (PÉREZ MELLADO, 1983) en el que se tiene en cuenta el número de individuos capturado en un mes, el número de muestreos realizados en el mismo y en el número total de muestreos:

$$
\operatorname{Im}=\frac{\mathrm{n}}{\% \mathrm{M}} ; \quad \quad \% \mathrm{M}=\frac{\mathrm{m}}{\mathrm{M}} \times 100
$$

Im: Indice mensual.

$\mathrm{n}$ : Número de individuos de la especie $\mathrm{Y}$ capturados en un mes.

\%M: Porcentaje de muestreo en el mes respecto al número total de muestreos.

m: Número de muestreos positivos de la especie $\mathrm{Y}$ en un mes.

M: Número total de muestreos.

La suma de los índices mensuales nos da un índice anual (Ia). El porcentaje del índice mensual respecto del anual nos da unos valores de actividad mensual corregida, referidos al número de individuos capturados de cada especie en cada mes:

$$
\frac{\operatorname{Im}}{\mathrm{Ia}} \times 100
$$

Para estudiar la distribución altitudinal se han delimitado 12 intervalos, de $100 \mathrm{~m}$. cada uno, constituyendo así 12 clases de altitud, entre las cuales se reparten las diferentes localidades muestreadas.

$\begin{array}{cc}\text { Intervalos de altitud } & \text { N. }{ }^{\circ} \text { de localidades } \\ 1.400-1.499 & 1 \\ 1.300-1.399 & 1 \\ 1.200-1.299 & 2 \\ 1.100-1.199 & 1 \\ 1.000-1.099 & - \\ 900-999 & 1 \\ 800-899 & 2 \\ 700-799 & 8 \\ 600-699 & 15 \\ 500-599 & 9 \\ 400-499 & 26 \\ 300-399 & 28 \\ 200-299 & 4\end{array}$


Para realizar el cálculo de la distribución altitudinal de aquellas especies que se disponía de un número representativo de capturas, se ha utilizado el siguiente índice de frecuencias corregidas (DAGET, 1972):

$$
F e(K)=\frac{U(K)}{R(K)} / \frac{U(E)}{N R}=\frac{U(K) \cdot N ~ R}{R(K) \cdot U(E)}
$$

$\mathrm{Fe}(\mathrm{K})$ : Frecuencia corregida de la especie $\mathrm{E}$ en la clase $\mathrm{K}$. cie $\mathrm{E}$.

U (K): Número de muestras de la clase $K$ en los que está presente la espe-

$R(K)$ : Número de muestras en la clase $K$.

$U(E)$ : Número de muestreos en los cuales la especie $E$ está presente.

N R: Número total de muestreos.

A continuación se indican aquellas localidades donde han sido encontrados ejemplares de la familia Geotrupidae, señalándose en cada una de ellas la altitud y las coordenadas UTM. El número que se indica delante de cada una de estas localidades corresponde a la ordenación general de las 98 localidades y que se encuentran reseñadas en el mapa 2:

\begin{tabular}{|c|c|c|}
\hline LOCALIDADES & $\begin{array}{c}\text { AlTITUD } \\
\text { (metros) }\end{array}$ & $\begin{array}{l}\text { COORDENADAS } \\
\text { U. T. M. }\end{array}$ \\
\hline 1. Abadía & 500 & 30TTK5761 \\
\hline 2. Acebo ........................... & 550 & 29TPE9753 \\
\hline 9. Baños de Montemayor & 700 & 30TTK556 \\
\hline 11. Bohonal de Ibor .......... & 300 & 30STK8203 \\
\hline 12. Cabezabellosa ............... & 650 & 30TQE5546 \\
\hline \multicolumn{3}{|l|}{ 13. Cabezabellosa (Pico } \\
\hline Torno) & 950 & 30TTK4546 \\
\hline 15. Cáceres. & 400 & 29SQD2673 \\
\hline 15. Cadalso ........ & 500 & 30TQE0957 \\
\hline 18. Cañameros ...... & 600 & 30STJ9561 \\
\hline 19. Carcaboso ...................... & 300 & 29TQE3836 \\
\hline 24. Cilleros ........................... & 450 & 29TPE8842 \\
\hline 26. Descargamaría ............ & 500 & 29TQE1364 \\
\hline 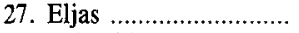 & 600 & 29TPE8453 \\
\hline 33. Grimaldo ....................... & 350 & 29TQE4662 \\
\hline 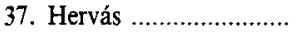 & 600 & 30TTK5661 \\
\hline 39. Hinojal ........................ & 350 & 29SQD2799 \\
\hline 42. Jaráiz de la Vera ........ & 450 & 30TTK 6638 \\
\hline 43. Jarandilla ……............... & 600 & 30TTK 7445 \\
\hline 44. Jerte & 600 & 30TTK6355 \\
\hline Jerte & 900 & 30TTK5955 \\
\hline Jerte & 1.200 & 30TTK5956 \\
\hline 46. La Garganta ................. & 1.300 & 30TTK6368 \\
\hline $\begin{array}{l}\text { 50. Losar de la Vera (G. de } \\
\text { Cuartos) }\end{array}$ & 400 & \\
\hline & 400 & HAg \\
\hline
\end{tabular}

\begin{tabular}{ccc}
\hline LOCALIDADES & $\begin{array}{c}\text { ALTITUD } \\
\text { (metros) }\end{array}$ & $\begin{array}{c}\text { COORDENADAS } \\
\text { U. T. M. }\end{array}$ \\
\hline
\end{tabular}

51. Madrigal de la Vera (G. de Minchones) ............

52. Malpartida de Plasencia

58. Navalmillar de Ibor ...

$350 \quad 30 T T K 9947$

$400 \quad$ 29SQD5329

59. Navalmoral de la Mata

61. Nuñomoral

$600 \quad 30$ STJ9384

$300 \quad 30$ STK8319

$\begin{array}{lll} & 600 & 29 T Q . . .\end{array}$

63. Pantano de Borbollón $350 \quad$ 29TQE0644

65. Pedroso de Acín ....... 400 29SQE2213

66. Pescueza ................... $350 \quad$ 29SQE0121

68. Piornal ..................... $1.250 \quad$ 30TTK6145

69. Plasencia ................... $350 \quad 30$ TTK 4440

72. Puerto de Honduras ... $1.450 \quad 30$ TTK5656

73. Robledillo de la Vera ... $400 \quad 30$ TTK 7942

74. Robledollano ............. $650 \quad 30$ STJ8587

75. San Martín de Trevejo $800 \quad$ 29TPE8754

77. Santibáñez el Alto ..... $600 \quad 29 T Q E 0852$

78. Santibáñez el Bajo ..... $350 \quad$ 29TQE3751

83. Tornavacas ............... $1.150 \quad$ 30TTK7361

86. Torrejón el Rubio .... $\quad 300 \quad$ 29SQE5806

88. Trevejo ..................... $700 \quad$ 29TQE8949

89. Trujillo ....................... $500 \quad 30$ STJ5271

92. Valverde del Fresno ... $500 \quad$ 29TPE8056

94. Villanueva de la Vera $400 \quad$ 30TTK9145

96. Zarza de Granadilla ... $350 \quad$ 29TQE4861 


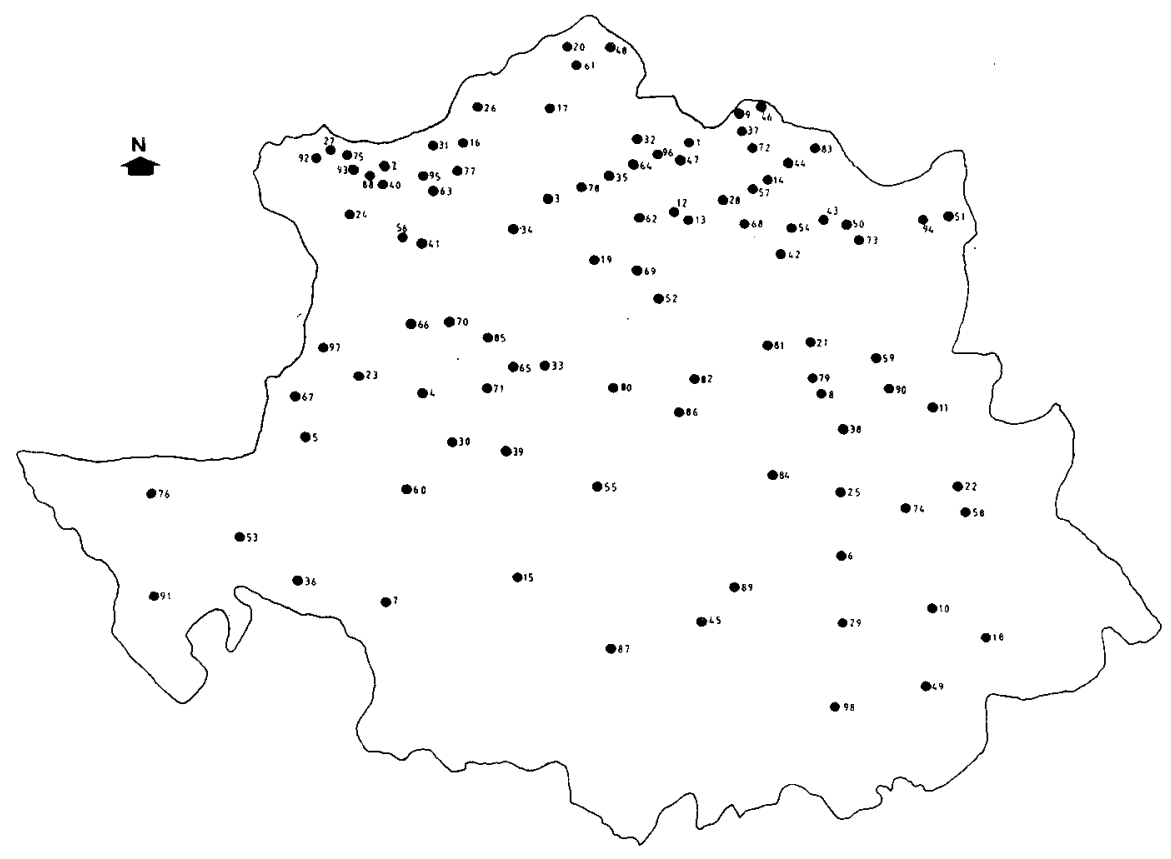

Mapa 2: Localidades prospectadas en el área de estudio.

Ceratophyus hoffmannseggi Fairmaire, 1856.

Distribución: Península Ibérica y Marruecos.

Hasta la actualidad las citas más septentrionales de esta especie en la $\mathrm{Pe}$ nínsula Ibérica son Burgos y Valladolid (BAGUENA, 1967; GALANTE, 1983).

Se han estudiado un total de 23 ejemplares de los que 11 se han encontrado en heces de vacuno, 1 en excremento de ovino y 3 fueron atraídos mediante un foco de luz. El número de capturas ha sido escaso aunque es una especie que parece encontrarse bien distribuida por toda la provincia. Se ha capturado en las siguientes localidades: Bohonal de Ibor, Cáceres, Carcaboso, Hervás, Navalmoral de la Mata, Pedroso de Acín, Pescueza, Trujillo y Zarza de Granadilla (Mapa 3). En todos los casos aparecen en altitudes inferiores a los 600 m., en áreas de clara influencia mediterránea en el dominio del encinar (Quercus rotundifolia Lam.) (Gráfica 1).

Los imagos se han encontrado desde finales del invierno (Marzo) hasta mediados de la primavera (Mayo), detectándose en el otoño otro período de actividad. Si bien el número de ejemplares capturados no permite confeccionar la gráfica de fenología, se observa que los datos obtenidos están de acuerdo con los aportados por GALANTE, 1981 en el área situada al Norte de las sierras de Gata y Peña de Francia (Provincia de Salamanca). 


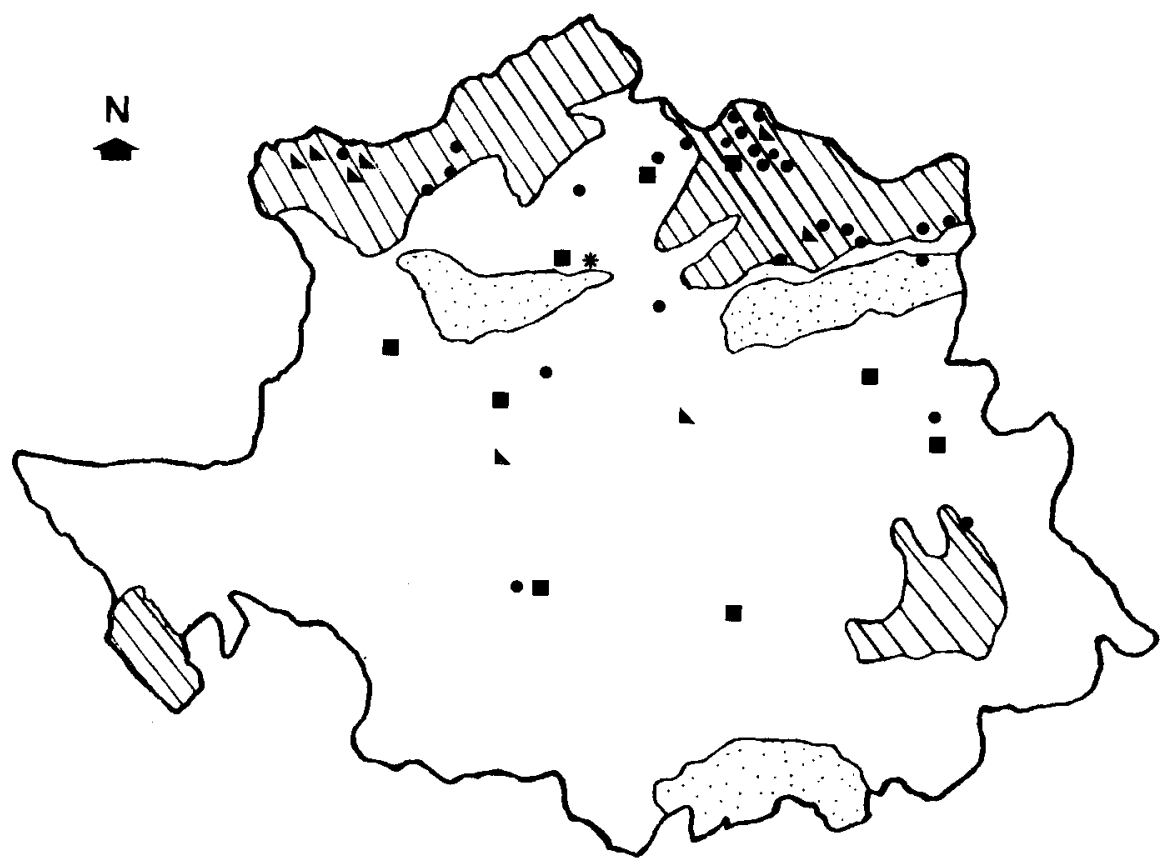

Mapa 3: Distribución geográfica de: Typhoeus typhoeus (L.). Thorectes nitidus Jekel. * Trypocopris pyrenaeus Charpentier. - Ceratohyus hoffmannseggi Fairmaire.

Typhoeus typhoeus (Linné, 1758)

Distribución: Europa occidental hasta Suecia y Norte de Marruecos.

El total de ejemplares estudiados fue 120 , de los cuales 46 se colectaron en excrementos de ovino, 26 en heces de equino, 21 en excrementos de caprino, 13 en excrementos de vacuno, 12 en heces de conejo y 1 en excremento humano. Esta especie parece ser más abundante en heces con bajo contenido hídrico como las de ovino y caprino.

La mayor parte de las localidades donde ha sido encontrada esta especie, se sitúan en el Norte de la provincia (Sierra de Gata, Montes Tras la Sierra y cara Sur de Gredos). Las localidades donde se ha efectuado capturas de la misma son: Abadía, Baños de Montemayor, Cáceres, Cadalso, Collado, Hervás, Jaraíz de la Vera, Jarandilla, Jerte 1.100 m., La Garganta, Losar de la Vera, Madrigal de la Vera (Garganta de Minchones), Malpartida de Plasencia, Navalmillar de Ibor, Pantano de Borbollón, Puerto de Honduras, Robledillo de la Vera, Robledollano, San Martín de Trevejo, Santibáñez el Alto, Santibáñez el Bajo, Villanueva de la Vera y Zarza de Granadilla. (Mapa 3).

Esta especie se ha recogido en altitudes que oscilan entre los $400 \mathrm{~m}$. y los $1.500 \mathrm{M}$. (Gráfica 2) en puntos donde la vegetación predominante es arbórea o arbustiva. Su área de distribución nos indica que es más abundante en la mitad Norte del área estudiada. 


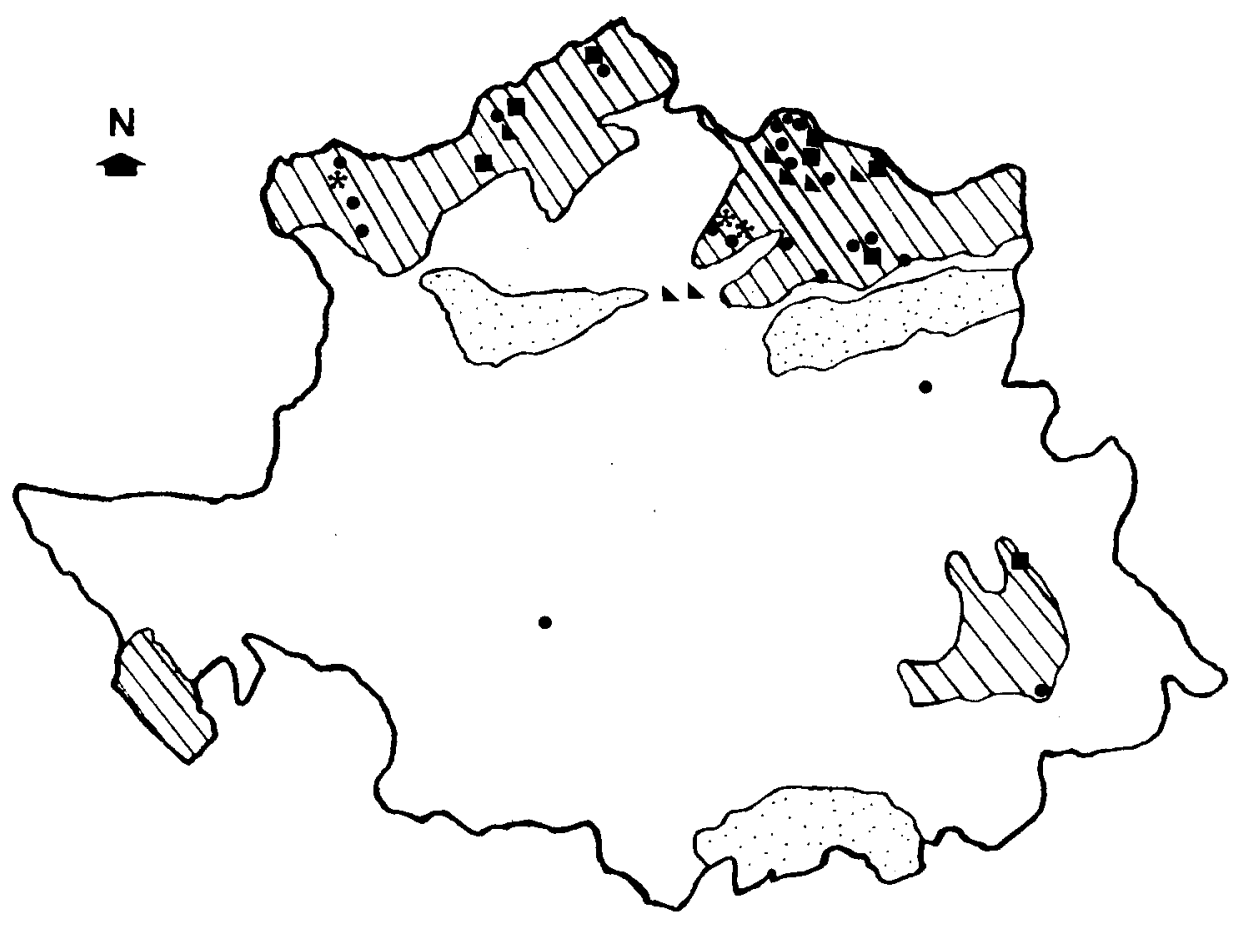

Mapa 4: Distribución geográfica de: Geotrupes mutator Marsham. * Geotrupes niger Marsham. Thorectes escorialensis Jekel. Geotrupes ibericus Baraud.

Los adultos aparecen en otoño y están presentes hasta mediada la primavera, como se deduce de la gráfica de fenología (Gráfica 4), estando estos datos de acuerdo con los aportados por BRUSSAARD, 1983.

Geotrupes mutator Marshan, 1802.

Distribución: Europa hasta el Cáucaso.

Se han estudiado 15 ejemplares de los cuales 4 se colectaron en excrementos de vacuno y 8 en heces de equino, excremento este último por el que muestra preferencia esta especie según PAULIAN y BARAUD (1982).

De las capturas realizadas, la mayor parte se localizan en altitudes superiores a los $1.000 \mathrm{~m}$. y en un área determinada (Montes Tras la Sierra) correspondiendo a las localidades de: Hervás, Jerte, Plasencia, Puerto de Honduras y Tornavacas. Sólo en un caso se colectó esta especie más al Oeste, en Descargamaría (Mapa 4). En las dos zonas la influencia atlántica es patente y la vegetación predominante son los piornales Cytision purgantis $\mathrm{R}$. Tx. en altitudes superiores a los $1400 \mathrm{~m}$. y el roble melojo (Quercus pyrenaica Will.) por debajo de los mismos. 

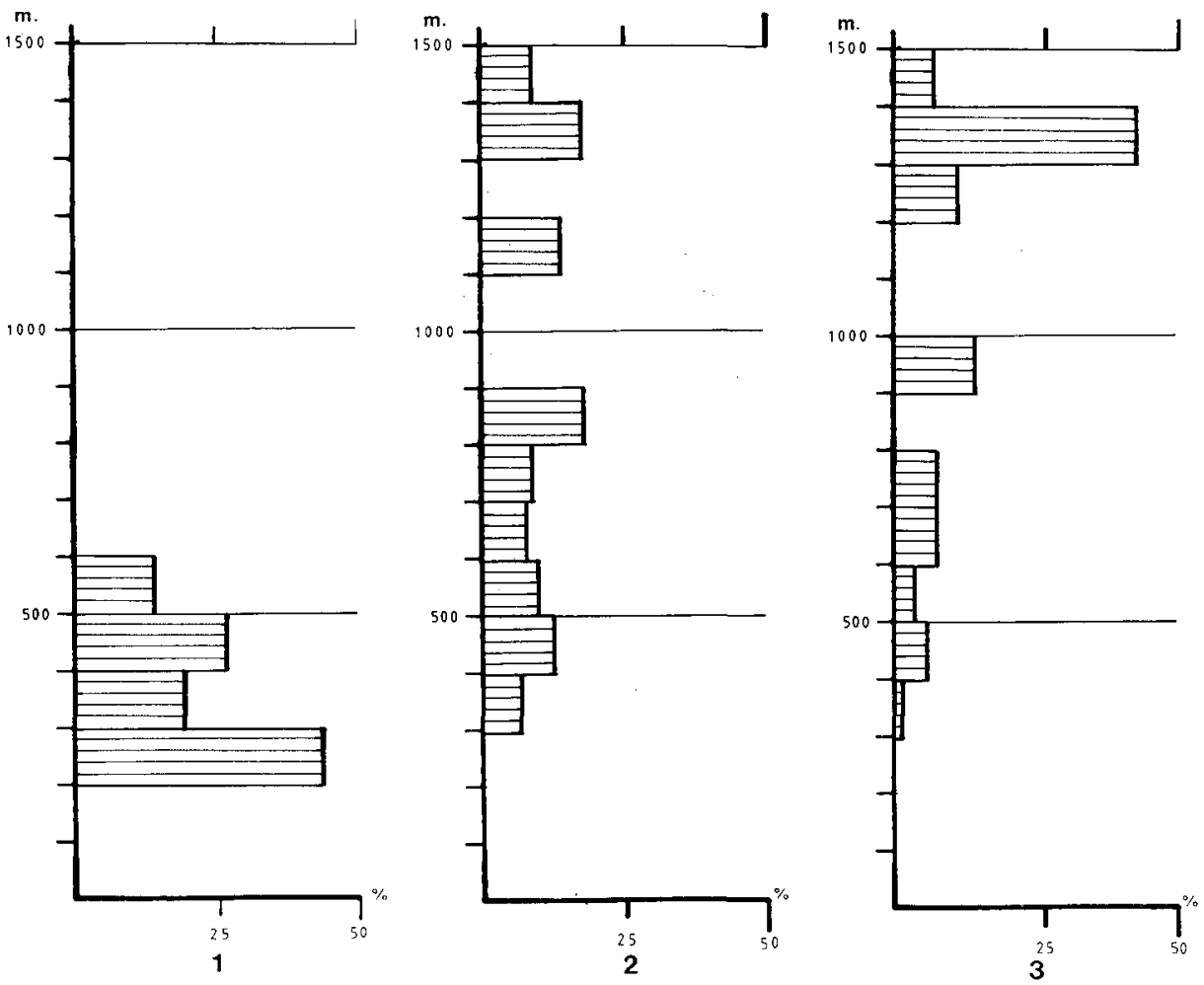

Distribución altitudinal de: 1.-Ceratophyus hoffmannseggi Fairmaire. 2.-Typhoeus typhoeus (L.). 3.-Geotrupes ibericus Baraud.

Los adultos se han recogido desde el otoño hasta la primavera, período durante el cual presentan actividad los imagos según PAULIAN 1959. Tan sólo un ejemplar fue encontrado en el mes de julio, época en la que pueden aparecer esporádicamente, como indica GALANTE (1981).

Geotrupes niger Marshan, 1802.

Distribución: Europa Occidental y parte occidental del Norte de África.

Se colectaron 5 ejemplares en heces de equino, 10 en heces de vacuno y 2 en heces de perro.

Como la especie anterior, se localiza en las sierras más septentrionales de la provincia, Montes Tras la Sierra y Sierra de Gata. Estas sierras están sometidas a una clara influencia oceánica, lo cual está en concordancia con lo expuesto por LUMARET (1979) que la considera como una especie ligada a zonas de montaña con influencia atlántica. Ha sido capturada en las siguientes localidades: Cabezabellosa, Cabezabellosa (Pico Torno), Eljas, Jarandilla, Jerte y Puerto Honduras (Mapa 4).

Es una especie cuyos imagos presentan actividad desde comienzos del verano (junio) hasta mediados el otoño (noviembre) de acuerdo con los datos obtenidos. 

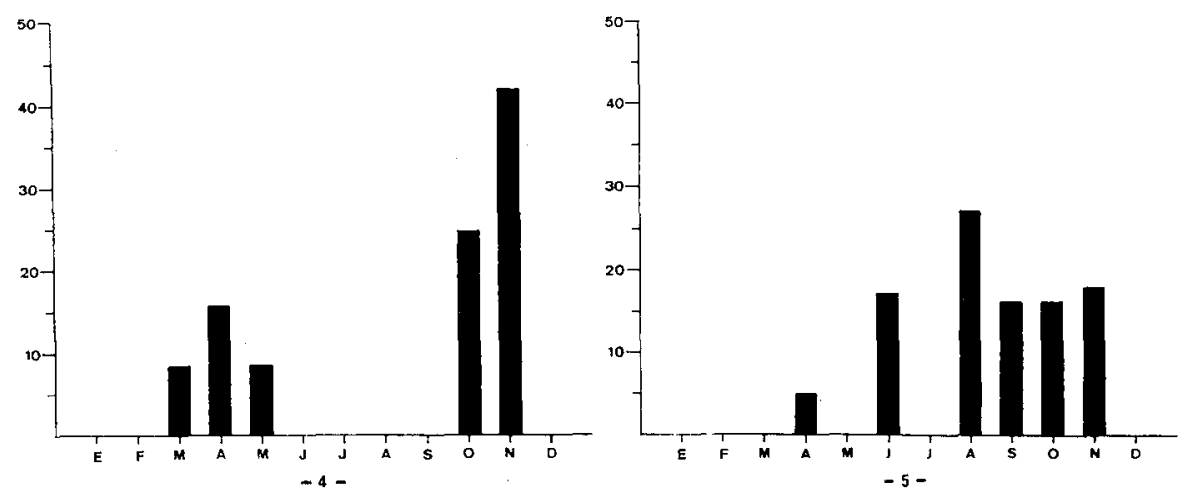

Actividad anual de: 4.-Typhoeus typhoeus (L.). 5.-Geotrupes ibericus Baraud.

Geotrupes ibericus Baraud, 1958.

Distribución: Península Ibérica.

Se colectaron 74 ejemplares en heces de vacuno, 25 en excrementos de equino y 1 en excrementos de perro.

Esta especie que parece ser más abundante en la parte septentrional de la provincia, también se ha capturado en el Sur y Sureste de la misma. Las localidades donde se han recogido fueron: Baños de Montemayor, Cabezabellosa, Cabezabellosa (Pico Torno), Cáceres, Cañameros, Cilleros, Descargamaría, Eljas, Jaraiz de la Vera, Jarandilla, Jerte, La Garganta, Navalmoral de la Mata, Nuñomoral, Piornal, Puerto de Honduras, Robledillo de la Vera (Mapa 4).

El intervalo altitudinal en el que se ha capturado es amplio, desde los 400 $\mathrm{m}$. hasta los $1.500 \mathrm{~m}$., lo cual confirma los datos aportados por BARAUD $(1958,1977)$, BOGGETTI et ZUNINO (1977) en cuanto al habitat de la especie que se diferencia del G. spiniger Marsh., en que ésta vive en altitudes no elevadas.

Como se observa en la gráfica de fenología los imagos se encuentran desde agosto hasta noviembre, período durante el cual se realiza la puesta (Gráfica 5). Los individuos que aparecen en primavera, aunque en bajo número, son probablemente individuos invernantes, y los ejemplares que se encuentran a principios del verano proceden probablemente de los huevos que eclosionaron antes del invierno, de acuerdo con BAGUENA (1959).

Thorectes escorialensis Jekel, 1865.

Distribución dentro de la Península Ibérica presenta una típica distribución atlántica: Sistema Central, Norte de Portugal y Galicia (GALANTE, 1984).

Durante la realización de este trabajo fueron colectados 30 ejemplares, de los cuales 24 fueron encontrados en heces de cabra.

Se ha capturado en las siguientes localidades: Cadalso, Casares de las Hurdes 1.020 m., La Garganta, Jarandilla, Navalmillar de Ibor, Puerto de Honduras y Tornavacas. 
De acuerdo con GALANTE (op. cit.) es una especie que se encuentra únicamente en las áreas de influencia atlántica (Mapa 4), como son las sierras ubicadas al Norte de la provincia de Cáceres. La única captura realizada al sureste del área de estudio (Navalmillar de Ibor) se localiza también en una zona de influencia oceánica que corresponde a la Sierra de Guadalupe y Sierra de la Palomera.

Thorectes nitidus Jekel, 1865.

Distribución: Península Ibérica.

Se han estudiado un total de 13 ejemplares de los cuales 8 fueron recolectados en excrementos de ovino. Se ha podido observar que el modo de trasladar el excremento de oveja hasta la galería es similar al descrito por LÓPEZ COLÓN (1985) para esta especie, así como el descrito por LUMARET (1978) para $T$. albarracinus Wagner. Los individuos sujetan con las tibias, la cabeza y los tarsos anteriores la hez, mientras que con las patas posteriores se apoyan en el suelo y caminan hacia atrás.

Parece ser una especie que se encuentra en el Noroeste del área de estudio, aunque también se ha colectado en el centro y Norte de la misma (Mapa 3). Las localidades donde se ha capturado son las siguientes: Acebo, Hervás, Hinojal, San Martín de Trevejo, Torrejón el Rubio, Trevejo y Valverde del Fresno.

No se puede relacionar con ningún dominio de vegetación, ya que aparece en áreas de influencia atlántica donde la vegetación predominante son los brezales como etapa de sustitución de los robledales (San Martín de Trevejo, Trevejo, Valverde del Fresno) y en zonas de encinares de clara influencia mediterránea (Torrejòn el Rubio). En ningún caso sobrepasan los $800 \mathrm{~m}$. de altitud. Trypocopris pyrenaeus Charpentier, 1825

Distribución: Europa occidental.

Se ha estudiado un macho capturado en Carcaboso el 3-IV-85 (P. Conejero Leg.) depositado en el Departamento de Zoología de la Universidad de Salamanca.

Durante la realización de este trabajo no se ha capturado esta especie, si bien ya había sido citada de Cáceres por BAGUENA (1967), lo cual induce a suponer que es una muy especie escasa y esporádica en el área de estudio.

\section{DISCUSIÓN}

Todas las especies estudiadas tienen como características común la preferencia por habitats cerrados que posean un adecuado dosel arbóreo o bien un matorral de porte elevado resultado de la aparición de etapas de sustitución de encinar o robledal. No obstante, en ocasiones, algunas especies como Geotrupes mutator Marshan pueden aparecer en pastizales abiertos.

Dentro de la comunidad de Geotrupidae de la zona prospectada, encontramos dos especies, Ceratophyus hoffmannseggi Fairmaire y Thorectes nitidus Jekel, que se distribuyen en áreas de poca altitud. En particular la especie beticorrifeña $C$. hoffmannseggi Fairm. es mucho más frecuente entre $300 \mathrm{~m}$. y $400 \mathrm{~m}$., no sobrepasando los $600 \mathrm{~m}$., mientras el elemento ibérico Th. nitidus Jek, sin superar los $800 \mathrm{~m}$., tiene como intervalo altitudinal preferente de 500 a $700 \mathrm{~m}$. Al contrario, la especie paleártica occidental G. niger Marsh. y el 
elemento lusitánico Th. escorialensis Jek., se han encontrado siempre por encima de los $600 \mathrm{~m}$. de altitud, pudiendo ser considerada dentro del área de estudio como propias de zonas montañosas. Así mismo encontramos dos especies que están presentes desde los 350 a los $1.450 \mathrm{~m}$., y son el endemismo ibérico G. ibericus Bar. y la especie paleártica occidental $T$. typhoeus (L.).

Por último la especie europea $G$. mutator Marsh., si bien ha sido localizada a $350 \mathrm{~m}$. de altitud, es más frecuente a partir de los $800 \mathrm{~m}$., llegando a los $1.450 \mathrm{~m}$., siendo ésta una de las cotas más elevadas del área.

Dentro de la distribución que presentan las diferentes especies, encontramos que $C$. hoffmannseggi Fairm. se localiza únicamente en las áreas fitoclimáticas de $Q$. rotundifolia Lám. que coincide con las cotas de menor altitud de la provincia de Cáceres.

Localizadas únicamente en el área fitoclimática de $Q$. pyrenaica Will., están presentes tres especies: G. mutator Marsh., G. niger Marsh., Th. escorialensis Jek.

Existe por otra parte un conjunto de otras tres especies formado por $T$. typhoeus (L. ), G. ibericus Bar., Th. nitidus Jek. que aparecen tanto en las áreas de encinar ( $Q$. rotundifolia Lam.) como de robledal ( $Q$. pyrenaica Will.); si bien son más abundantes en esta última.

Se observa en general una mayor concentración de todas las especies del género Geotrupes y Thorectes en el tercio Norte de la provincia de Cáceres, coincidiendo en general con las estribaciones montañosas y sus áreas adyacentes, que en gran parte están incluidas en el área fitoclimática de $Q$. pyrenaica Will. Vemos así mismo que $T$. typhoeus (L.) presenta una mayor tendencia a la concentración en localidades de la mitad Norte del área estudiada.

Esta distribución viene condicionada por los requerimientos de las diferentes especies. De acuerdo con LUMARET (1978) G. mutator Marsh. y T. typhoeus (L.) necesita un cierto grado de humedad y un suelo que permita la elaboración de galerías más o menos profundas, para lo cual el terreno debe estar capacitado para la retención de agua. Esta es probablemente la causa de que ambas especies se encuentren dentro de un amplio intervalo altitudinal. En áreas de baja altitud estas dos especies se localizan donde el matorral suele ser espeso o bien existe un dosel arbóreo propicio que concentre la humedad. Así mismo ambas especies penetran ampliamente en las sierras, donde son más abundantes, ya que es en estas zonas donde se da el grado de humedad y profundidad del suelo más acorde con las necesidades de las mismas.

Probablemente el endemismo ibérico G. ibericus Bar. tenga unos requerimientos semejantes a los mencionados, de ahi que aparezca dentro de un amplio espectro altitudinal y tanto en áreas fitoclimáticas de encinar como de robledal. Al igual que la especie europea $G$. spiniger Marsh., muestra preferencia por terrenos de cierta humedad y posibilidad de excavación profunda, si bien se diferencia por el rango altitudinal que colonizan.

La provincia de Cáceres está constituida en su mayor parte por una gran penillanura, cuya altitud media se sitúa alrededor de los $400 \mathrm{~m}$. Estos terrenos abiertos, con influencia mediterránea, cubiertos en parte por un dosel arbóreo de encinar ( $Q$. rotundifolia Lam.) están sometidos a una gran sequía durante casi todo el año. Probablemente ésta es la causa de que la mayor parte de las especies se encuentren acantonadas en el Norte de la provincia, en las sierras 
y áreas limítrofes, que coinciden con las zonas de influencia atlántica (Zona fitoclimática de $Q$. pyrenaica Will.).

La única especie que claramente vive en terrenos secos, si bien aparece en pastos con vegetación arbórea o arbustiva, no penetrando en el área fitoclimática de $Q$. pyrenaica Will., es la especie beticorrifeña $C$. hoffmannseggi Fairm.

Por todo esto podemos concluir que en el Sistema Central se establece el límite de distribución de alguna de las especies estudiadas. Parece constituir el extremo meridional de distribución en el Oeste de la Península Ibérica, de G. mutator Marsh., G. niger Marsh., Th. escorialensis Jek. Esta última es una especie de clara distribución atlántica que se encuentra en toda Galicia y mitad Norte de Portugal, penetrando en el interior de la Península Ibérica a través del Sistema Central (GALANTE, 1984).

También en el caso de C. hoffmannseggi Fairm., parece que en el Sistema Central coincide aproximadamente su límite de distribución septentrional en el Oeste peninsular, si bien puede encontrarse al Norte de esta barrera natural (GALANTE, 1981, 1983), no obstante es escasa en estas áreas.

Este conjunto de especies puede llegar a tener una eficaz actuación en la degradación de excrementos del ganado, a excepción de Th. escorialensis Jek. y Th. nitidus Jek. que se alimentan de heces con muy bajo contenido hídrico como son las de oveja y cabra fundamentalmente, el resto de las especies acuden a otro tipo de excremento vacuno, equino u ovino. En concreto las diversas especies de Geotrupes muestras claras preferencias por las heces de vacuno o equino, mientras $T$. typhoeus (L.) es atraído con mayor eficacia por las de ovino y equino.

Como ya se indicó anteriormente, dada la sequía imperante en la provincia de Cáceres durante toda la época estival, es práctica habitual durante este período el traslado del ganado ovino y vacuno fundamentalmente a las montañas del centro y Norte de la Península Ibérica. En la provincia de Cáceres durante el verano únicamente quedará ganado en algunas partes de las sierras.

Si examinamos la fenología de las diferentes especies observaremos que todas ellas son activas durante el otoño, época del regreso del ganado. Así mismo durante la primavera presenta una gran actividad $C$. hoffmannseggi Fairm. en áreas de encinar y G. Mutator Marsh. en áreas de robledal, actuando ambas sobre excrementos de ganado vacuno y equino. También curante la primavera tiene importancia $T$. typhoeus (L.), si bien actúa tanto en áreas de encinar como robledal y fundamentalmente sobre las heces de ovino.

Ninguna de estas especies presenta actividad estival, al igual que ocurre en otras áreas de la Península Ibérica (GALANTE, 1981). En el verano se inicia la actividad de los imagos de G. ibericus Bar. y G. niger Marsh., si bien, en ocasiones, puede encontrarse algún ejemplar aislado en primavera probablemente invernante del año anterior. Estas dos especies actúan principalmente sobre las heces de ganado vacuno y equino y durante el verano se encuentran lógicamente en las zonas de pastoreo de las sierras cacereñas.

También durante el verano encontramos Th. escorialensis Jek. y Th. nitidus Jek., si bien la incidencia de estas dos especies en la destrucción de excrementos es mínima, dado sus hábitos alimenticios. Por otra parte, Th. escorialensis Jek. permanece activo gran parte del año. 
Vemos, por tanto, que se establece una gradación anual en la comunidad de Geotrupidae de la provincia de Cáceres, aunque en conjunto pueden ser considerados como coleópteros coprófagos típicos del otoño.

\section{APÉNDICE}

A continuación, se indican las fechas, localidades y número de ejemplares capturados de cada una de las especies estudiadas. La localidad viene determinada por el número de orden que se señala en la relación de las mismas.

\section{C. hoffamannseggi Fairm.}

11: 5-IV-83, 1 h (*). 15: abril-76, $1 \mathrm{~m} \mathrm{(*).} \mathrm{19:} \mathrm{3-IV-85,} 1$ h. 37: 19-III-82, 1 h; 12-IV-83, 1 m, 2 h. 59: 16-V-76, 1 h. 65: 26-XI-85, 4 m, 5 h. 66: 26-XI-85, $1 \mathrm{~m}, 1$ h. 89: 17-IV-83, $1 \mathrm{~m}, 2$ h. 96: 30-XI-84, 1 h.

(*) $m$ significa macho, y $h$, hembra.

\section{T. typhoeus (L.)}

1: 30-XI-84, 1 h. 9: 30-IV-83, 1 m. 15: IV-76, 1 h. 16: 24-IV-82, 1 h. 33: 26-XI-85, $2 \mathrm{~m}, 1$ h. 37: 21-III-82, 1 m. 42: 8-V-82, 1 h; 28-XI-84, 3 m. 43: 15-IV-82, $4 \mathrm{~m}, 1 \mathrm{~h} ; 28-\mathrm{XI}-85,17 \mathrm{~m}, 15$ h. 44 (1.100 m.): 17-V-80, $1 \mathrm{~h} ; 12-\mathrm{V}-84$, 1 h. 46: 28-X-80, 1 m, 1 h. 51: 29-XI-85, 1 m, 1 h. 52: 28-XI-84, 1 h. 58: 29XI-84, $6 \mathrm{~m}, 4$ h. 63: 11-IV-83, 1 m. 72: 28-IV-80, 1 m. 73: 28-XI-85, $3 \mathrm{~m}, 1$ h. 74: 29-XI-84, $15 \mathrm{~m}, 14$ h. 75: 7-III-81, 1 m. 77: 16-IV-82, 1 m. 78: 11-IV-83, 1 m. 94: 29-XI-85, 2 m, 2 h. 96: 30-XI-84, 1 h.

\section{G. mutator Marsh.}

26: 27-IX-80, 1 m. 37: 19-IV-82, 1 m. 44 (1.200 m.): 29-IX-80, 1 h. 69: 1-V-82. 1 h. 72: 17-V-80, 1h; 28-X-80, $4 \mathrm{~m}, 1$ h; 4-VII-82, 1 h. 83: 1-X-82, $2 \mathrm{~m}, 2 \mathrm{~h}$.

\section{G. niger Marsh.}

12: 27-VI-80, $2 \mathrm{~m}, 1$ h. 13: 14-VIII-84. 1 m. 27: 26-IX-80, 1 h. 43: 28-XI85,1 m. 44 (600 m.): 25-VIII-80, $1 \mathrm{~m}, 7$ h; 27-VI-80, 2 h. 72: 4-VII-82, 1 h.

\section{G. ibericus Bar.}

9: 16-VIII-84, $3 \mathrm{~m}, 4$ h. 12: 27-VI-80, $5 \mathrm{~m}, 1$ h. 13: 16-VIII- $84,9 \mathrm{~m}, 8$ h. 15: 20-VIII-83, 1 h. 18: 15-VIII-84, 1 m. 24: 12-IV-83, 1 m. 26: 27-IX-80, $1 \mathrm{~m}, 1$ h. 27: 26-IX-80, $4 \mathrm{~m}, 2$ h; 29-IX-80, 2 m, 3 h. 42: 28-XI-84, 6 m, 3 h. 43: 28-XI-85, 1 m. 44: 25-VIII-80, $3 \mathrm{~m}, 2$ h; 27-VI-80, 1 m. 46: 28-X-80-, $1 \mathrm{~h} ; 14-$ VIII-84, $15 \mathrm{~m}, 10 \mathrm{~h}$; 5-VIII-84, 1 h. 59: 15-VIII-84, $1 \mathrm{~m} .61: 28-I X-80$, 2 m. 68: 15-VIII-84, $2 \mathrm{~m}, 1$ h. 72: 28-X-80, $1 \mathrm{~m}, 3$ h. 73: 28-XI-85, 1 h. 
Th. escorialensis Jek.

16: 1 h. 43: 15-IV-82, 1 h. $46: 1 \mathrm{~m}, 2$ h. 58: 29-XI-84, 1 h. 72: 17-V-80, $2 \mathrm{~m}, 3 \mathrm{~h} ; 28-\mathrm{X}-80,1 \mathrm{~m}, 2 \mathrm{~h} ; 14-\mathrm{V}-82,1 \mathrm{~m}, 5 \mathrm{~h} ; 4-\mathrm{VII}-82,6$ h. 83: $1 \mathrm{~m}, 2 \mathrm{~h}$.

Th. nitidus Jek.

1: 7-II-82, 1 h. 37: 20-III-82, 1 m. 39: 27-XI-85, 3 m, 4 h. 75: 5-X-81, 1 m. 86: 27-VI-83, 1 m. 88: 22-IV-8, 1 m. 92: 7-II-85, 1 h.

Trypocopris pyrenaeus Charp.

19: 3-IV-85, $1 \mathrm{~m}$.

\section{AGRADECIMIENTO}

Queremos expresar nuestro sincero agradecimiento al doctor Enrique Rico Hernández por la elaboración del apartado de vegetación. 


\section{BIBLIOGRAFÍA}

ALLUE ANDRADE, J. (1966). Subregiones fitoclimáticas de España. Ministerio de Agricultura. Inst. Forestal de Invest. y Experim. Madrid, 57 p.

BÁGUENA CORELLÁ, L. (1959). Notas sobre ecologia y etología de los Scarabaeoidea ibéricos de interés en Edafología (Coleóptera). Graellsia XVII (1-3): 29-45.

- (1967). Scarabaeoidea de la fauna Ibero-Balear y Pirenaica. C.S.I.C. Madrid. 559 p.

BARAUD, J. (1958). Un nouveau Geotrupes Latr. d'Espagne. Soc. Ent. Lyon, 8: 2, 19-221.

- (1977). Coléoptères Scarabaeidae. Faune de l'Europe Occidentale. Belgique, France, Grande Bretagne, Itale, Peninsula Iberique. Publications de la Nouvelle Revue d'Entomologie. IV. Toulouse. $351 \mathrm{p}$.

BOGGETI, C. et ZUNINO, M. (1977). Il rango tassonomico e la distribuzione geografica de Geotrupes ibericus Baraud (Col. Scarabaeoidea). Boll. Soc. Entom. Italiana, 109 (1-3): 31-34.

BRUSSAARD, L. (1983). Reproductive behaviour and development of the dung beetle. Thyphoeus typhoeus (L.) (Col. Geotrupidae). Tijdschrift Voor Entomologie, 126 (10): 203-231.

DAGET, Ph. M., GUILLERM, J. L. (1972). Profils ecologiques et information mutuelle entre espéces et facteurs écologiques. Grand. Meth. Pflanz, Int. Symp. Veget. Rinteln 1970: 121-149.

FINCHER, G. T., HOWSON, W. G. and BURTON, G. W. (1981). Effects of cattle feces rapidly buried by dung beetles on yield and quality of Coastal Bermudoprass. Agronomy Journal, 73: 775-779.

GALANTE, E. (1981). Los Scarabaeoidea (Col.) de las heces de vacuno de la provincia de Salamanca. IV. Geotrupidae. Bol. Asoc. Esp. Entom. 4 (1980): 173-177.

- (1983). Sobre los escarabeidos (Col. Scarabeoidea) de la Península Ibérica (I). Bol. Asoc. Esp. Entom. 7: 55-68.

- (1984). Los Thorectes del grupo «escorialensis» en la Península Ibérica. (Col. Scarabaeoidea). Bol. Asoc. Esp. Entom. 8: 271-281.

GARCÍA RODRÍGUEZ, A., y FORTEZA BONNIN, J. (1970). Mapa de suelos de la provincia de Cáreces. C.S.I.C. Centro de Biología y Edafología Aplicada de Salamanca. 122 p.

LÓPEZ COLÓN, J. I. (1985). Contribución al género Thorectes Mulsant. VI. Las especies de la provincia de Madrid (Col. Geotrupidae). Nouv. Rev. Ent. (N. S.) 2 (2): 221-225.

LUMARET, J. P. (1978). Biogéographie et écologie des Scarabeides coprophages de sud de la France. Tesis Doctoral. Montpellier. Vol. I. 253 p.

- (1979). Biogéographie et écologie des Scarabeides coprophages de sud de la France. I. Méthodologie et modeles de repartition. Vie et Milieu, 1978-1979. Vol. XXVIII-XXIX, Fasc. 1, Ser. C: 1-34.

- (1979). Biogéographie et écologie des Scarabeides coprophages de sud de la France. II. Analyse synocologique des repartitions. Vie et Milieu, 1978-1979. Vol. XXVIII-XXIX, Facs. 2, Ser. C: $179-201$.

PAULIAN, R., y BARAUD, J. (1982). Faune des coléoptères de France. Lucanoidea et Scarabaeoidea. Editions Le Chevalier. Paris. 477 p.

PÉREZ MELLADO, V. (1983). Activity and thermoregulation patterns in two species of Lacertidae: Podarcis hispanica Stiendachner, 1870) and Podarcis bocagei. (1984). Cienc. Biol. Ecol. Syst. (Portugal), 5: 5-12. 